Urban Water Systems \& Floods II 143

\title{
AN INVESTIGATION OF SMALL AND MEDIUM-SIZED ENTERPRISES' FLOOD PREPARATION AND INSURANCE COVERAGE USING AGENT-BASED MODELLING
}

\author{
MESHAL ALHARBI ${ }^{1} \&$ GRAHAM COATES ${ }^{2}$ \\ ${ }^{1}$ Department of Computer Science, Durham University, UK \\ ${ }^{2}$ School of Engineering, Newcastle University, UK
}

\begin{abstract}
Flooding can result in significant financial losses to a nation's economy in part due to the damage and disruption caused to businesses. In the United Kingdom (UK), Small and Medium-sized Enterprises (SMEs) are crucial to the economy as they account for $99.9 \%$ of all businesses. In recognizing the economic importance of SMEs, and their limited resources and tendency to lack disaster mitigation plans, there is a need to investigate ways in which these businesses can improve in terms of flood preparation. An agent-based modelling approach is being developed to facilitate investigations into the effect of a range of precautions that SMEs can take in preparation for flooding. With a focus on manufacturing SMEs, agents representing these businesses have been modelled as having behaviours that can be enacted pre- and post-flood. Agent-based modelling and simulation (ABMS) has been used, coupled with a case study of the 2007 flood of the Lower Don Valley in Sheffield, to investigate the influence of different types of insurance coverage and financial status on the response and recovery of manufacturing SMEs exposed to different levels of flooding. As expected, results indicate that SMEs with comprehensive insurance and funds available, along with allocating employees to clean-up its premises, leads to a reduced recovery stage. However, in addition, results provide an indication of the degree of influence of combinations of these attributes on SME recovery.

Keywords: small and medium-sized enterprises, agent-based modelling and simulation, flooding, business recovery.
\end{abstract}

\section{INTRODUCTION}

Flooding is one of nature's most destructive events that causes damage to almost every sector of an economy [1], [2]. The UK's 5.4 million SMEs are essential to the country's economy as they account for $99.9 \%$ of all businesses [3]. In the UK, an SME is considered to be any business with fewer than 250 employees [4]. Furthermore, SMEs are categorized as micro-, small- or medium-sized according to employee numbers: a micro-sized business has 1-9 employees; a small business 10-49; and a medium-sized business 50-249 [5]. However, despite SMEs economic importance, in recent years this sector has proven vulnerable to extreme weather events [6], [7] due to their limited resources [8], [9] and their tendency not to implement disaster mitigation plans [10], [11]. Flooding, in particular, has caused significant financial losses to UK SMEs and remains a serious threat [12]. According to the Environment Agency (EA), UK businesses' losses due to flooding in 2007 were in the region of $£ 740$ million [13]. In England, it is projected that the annual loss suffered by residential and business properties due to flooding will exceed £1 billion [14]. Thus, mitigating the potential risk and disruption caused to SMEs by flooding has become an issue of key significance [15]. SMEs preparation for, response to and recovery from disruptive events including flooding - depend on a myriad of complex and interdependent processes [12]. Agent-based models (ABMs) - computational constructs used to simulate the actions and interactions of autonomous agents representing particular entities - are widely recognized as an effective tool for solving complex problems in business and the social sciences [16]-[19]. Indeed, it is well established that ABMs constitute a useful predictive and analytical research 
tool that provide insights into complex social behaviour and generate new theory [20]. As Chappin et al. [21] point out, while ABMs originate from research domains such as artificial intelligence and social science, they are flexible enough to be used in a wide range of applications, including disaster management. Clarke [22] indicated that ABMs are preferred when the goal is the simulation of a behavioural unit, such as a person or household, and when the model represents interactions among one or more types of agent. This paper investigates the effect of a range of combination of insurance coverage and financial status of SMEs on their recovery from flooding.

\section{MODELLING AND SIMULATION APPROACH}

The research reported in this paper builds on earlier work [2], [23]-[25] with a focus on the development and use of ABMS to investigate the effects on recovery of a range of flood related precautions and behaviours adopted by SMEs in the manufacturing sector. The approach involves linking a modelled geographical environment (MGE), flood event simulation and ABMS. The MGE consists of three layers of OS MasterMap ${ }^{\circledR}$, namely the Topography layer, Integrated Transport Network (ITN) layer, and AddressBase Plus layer [26]. Using these data layers enables a specific geographical area to be modelled in which the location of each individual business can be identified. In addition, within the MGE, flood event simulation generates inundation data at regular time intervals (half hour) such that the water depth at all locations throughout the area modelled, some of which coincide with the location of an SME's premises, is known. ABMS involves autonomous agents representing manufacturing SMEs exhibiting a range of pre- and post-flood behaviours and able to administer a number of potential flood-preparedness precautions. In addition to manufacturing SMEs, related organizations are modelled such as customers, suppliers, insurance companies, the Environment Agency (EA), cleaning service providers, electricians, plumbers, and IT specialists.

\section{AGENT-BASED MODELLING OF MANUFACTURING SMES}

\subsection{Overview}

Manufacturing SMEs are modelled as agents with behaviours, static attributes (e.g. name and size) and dynamic attributes - raw materials $(R M)$, machines $(M)$, employees $(E)$ and power $(P)$. As discussed in [24], SMEs' dynamic attributes change during the course of a flood event and contribute directly to performance. For example, in terms of a manufacturing SME's performance, in each half hour period (i.e. simulation tick, $t_{s}$ ), if the premises has power, the raw materials available exceeds or is equal to the amount to be used, the total of machines available to be used is equal to the total number of machines at the premises, and all production employees are available and work on production, then production level will be $100 \%$. However, should any of the dynamic attributes not be as stated, then production level will fall below $100 \%$.

Agent behaviours can be undertaken pre- and post-flood. Also, behaviours have a priority in terms of the order in which they should be undertaken and are classified according to whether a single or multiple employees is required to undertake them. Decisions to enact behaviours depends not only on their priority but also on (i) flood onset time and duration, and (ii) precautions implemented. Precautions are implemented by an SME prior to a flood event occurring and are intended to prevent or reduce water entering premises, and/or remove the need for (or reduce the duration of) particular behaviours. 
Pre- and post-flood, SMEs implement various allocation strategies to assign employees to work on behaviours depending on the situation at hand. Under normal (no-flood) conditions, an SME will allocate all of its available production employees to work on production. However, pre-flood, once an alert from the EA has been received, signalling a flood is likely to occur, an SME may allocate production employees to work on pre-flood behaviours while the remainder continue to work on production. EA alerts may arrive 36 hours prior to an expected flood occurring [27], [28], whereas EA warnings may be received up to three hours prior to an expected flood; SMEs trust EA warnings and so always act on them. Thus, once an EA warning has been received, all available production employees will be allocated to work on the behaviours to prepare for the expected flood event. Post-flood, i.e. in the recovery stage, production employees will be assigned to complete the behaviours required to be undertaken to return to normality, e.g. clean-up the premises. If possible, at the same time, some productions employees may be assigned to work on production.

\subsection{Pre-flood behaviours}

Employees work on production until an EA alert or warning is received or water enters the premises, in which case employees will evacuate. On receipt of an alert or warning, SMEs may allocate all or a proportion of production employees to enact all or some of the appropriate pre-flood behaviours, such as: placing sandbags at doorways; covering air-bricks; lifting-up IT equipment, documents, raw materials, light machines, and products. The aforementioned behaviours may be enacted simultaneously or sequentially based on (i) the number of production employees available, (ii) the number of scheduled pre-flood behaviours, (iii) the time at which the alert or warning is received, and (iv) the precautions implemented.

\subsection{Post-flood behaviours}

The post-flood period begins when the water recedes from company's premises. At this point, an SME performs the required post-flood behaviours to restore normal production. The term "normal production" refers to when an SME has resumed its pre-flood level of production. Thus, the power supply must be restored initially or repaired. For the latter, an electrician must attend the premises to carry out the necessary repairs, whereas the employees can do the former. Simultaneously or sequentially, SMEs may need to perform a range of necessary behaviours such as repairing sockets; contacting insurers (if insured) as well as plumbers, IT specialists, and cleaning service providers; allocating employees to clean-up premises; and returning the lifted-up items to their normal pre-flood positions.

\subsection{Precautions}

Precautions are implemented well in advance of a flood event taking place and are intended to prevent or reduce water entering the premises and/or remove the need for some behaviours or reduce the duration required to carry them out. For example, SMEs registered for EA alerts or warnings will be likely to prepare more effectively, by carrying out pre-flood behaviours, than those not registered. Similarly, insured SMEs, may be expected to recover at a faster rate than non-insured SMEs. In addition to the aforementioned precautions, the remainder of this set are intended to improve a company's level of preparedness; examples being settingup and maintaining an emergency financial reserve, installing flood-resilient flooring, maintaining an electricity generator on site, raising the location at which IT equipment is placed, maintaining sandbags on site, and installing flood barriers at doorways. 


\section{SME-RELATED ORGANIZATIONS}

SMEs interact with a range of organizations such as customers, suppliers, insurance companies, cleaning service providers, electricians, plumbers, and IT specialists. Post-flood, the process followed by an SME in relation to insurance is illustrated in Fig. 1.

Insured SMEs with comprehensive cover will receive assistance from a cleaning service provider in addition to receiving financial compensation. In contrast, SMEs with basic insurance cover will only receive the latter. As part of the SESAME project [29], according to interviews undertaken with SMEs having experienced flooding, some of these businesses chose to contact their insurer whereas others did not.

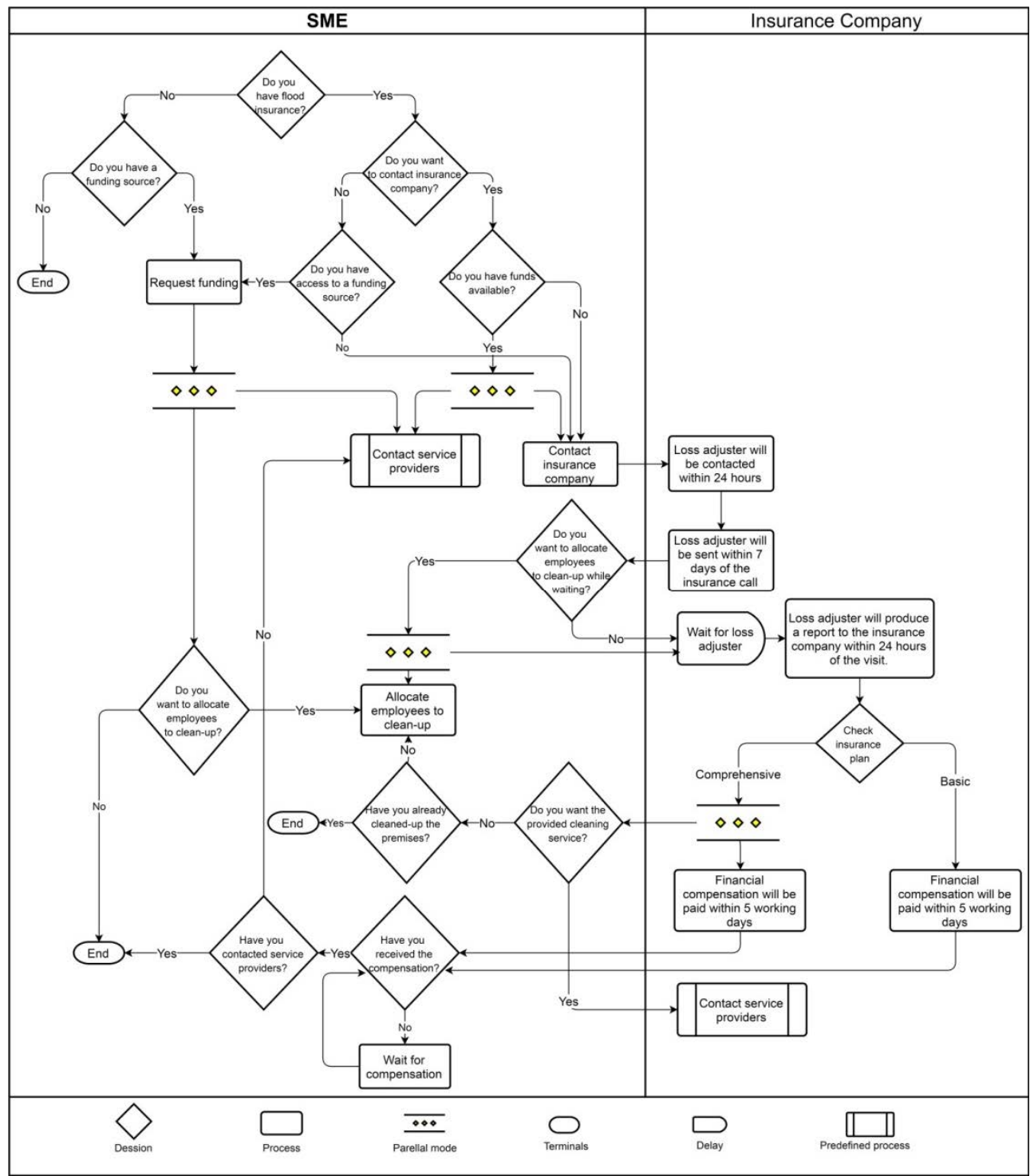

Figure 1: SMEs contacting insurance company. 
The reason for not doing so was to avoid a potential increase in their future insurance premium. However, in such cases, these SMEs needed sufficient funds to be in place to ensure business continuity. If the insurance company is contacted by an SME, a loss adjuster will be sent within seven days to assess the damage and produce a report [30] followed by payment being released within five working days of the visit [31]. During this potential 12day period, SMEs may choose to allocate their own employees to clean-up the premises rather than waiting for the cleaning service provided by the insurance to arrive. Alternatively, if sufficient funds are available, SMEs may themselves choose to contact service providers. In this research, service providers are modelled regionally and nationally in terms of their location. Further, national service providers are only contacted if an SME is unable to secure the services of a regional provider, which are limited in number.

\section{AGENT-BASED SIMULATIONS}

\subsection{Case study}

In this paper, the case study considered is related to the 2007 flood event in the Lower Don Valley region of Sheffield in the UK. As discussed in [24], this region was selected due to its high concentration of SMEs some of which have experienced flooding and/or are prone to flooding. The flood event simulation generated inundation data representing, the water depth throughout the region modelled at 30-minute intervals over a 45.5-hour period. Using the MGE of the region considered in which 1463 manufacturing SMEs were identified, coupled with the inundation data, 256 of these experienced flooding at some point during the 45.5hour event. Based on the statistical data related to businesses in the UK, $99.9 \%$ of all businesses are SMEs, with $96 \%, 3.3 \%$, and $0.6 \%$ categorized as micro-, small- and mediumsized, respectively [5]. Thus, in this research, the 256 flooded manufacturing SMEs were categorized as 246 being micro-sized, 8 small and 2 medium-sized.

Fig. 2 displays the MGE and flood footprint at hour 25 of the 45.5-hour event. Also, in Fig. 2, the locations of three micro-sized SMEs ("a", "b" and "c") are indicated in areas "A", "B" and " $C$ " in which the majority of SMEs experienced light, moderate and severe flooding respectively. Despite suffering different levels of flooding, these specific SMEs have been selected due to the flood water reaching and receding from their respective premises being the same. The SMEs highlighted will be referred to in Sections 5 and 6.

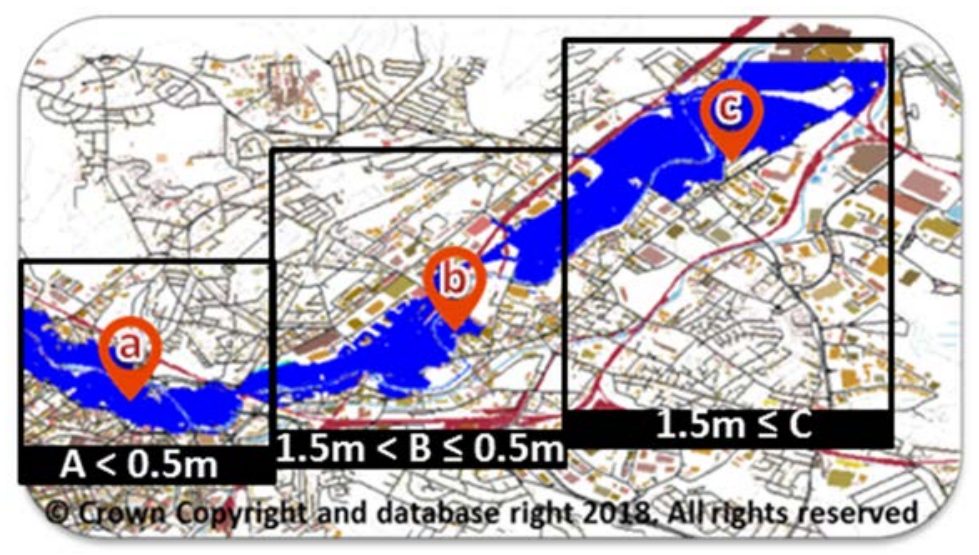

Figure 2: MGE and flood footprint at hour 25 of the simulated flood event. 
Table 1: Simulation experiments.

\begin{tabular}{|c|c|c|c|}
\hline Experiment & Insurance cover & Funds available & Clean-up by \\
\hline S1 & Basic & No & Employees \\
\hline S2 & Comprehensive & Yes & Service provider \\
\hline S3 & Comprehensive & Yes & Employees \\
\hline
\end{tabular}

\subsection{Experiments}

The investigation presented in this paper involves three simulation experiments with each representing different combinations of precautions implemented by an SME which, in turn, leads to different pre- and post-flood behaviours being undertaken. More specifically, with a focus in insurance coverage, the simulation experiments are defined in Table 1.

All three experiments were carried out for each micro-sized business indicated in Fig. 2 (labelled "a", "b" and "c") with each having the same number of employees for comparative purposes.

\section{RESULTS AND DISCUSSION}

In all simulation experiments undertaken, for each of the three micro-sized SMEs selected (see Fig. 2), EA alerts were received prior to the flood event taking place during working hours on day 2 from clock time $t_{c}=8: 00$ to $t_{c}=18: 00$, i.e. from simulation time $t_{s}=49$ to $t_{s}=69$. Thus, during this period, these businesses allocated their production employees to lift-up their IT equipment, documents, light machines, and products; and to cut-off the electricity supply to avoid damage. Subsequently, for SMEs "a", "b" and "c" the flood commenced at $t_{s}=119$ $\left(t_{c}=19: 00\right.$ on day 3$)$ and receded at $t_{s}=197\left(t_{c}=10: 00\right.$ on day 5$)$, reaching a depth in their respective premises, with no precautions implemented, of $0.38 \mathrm{~m}, 1.16 \mathrm{~m}$, and $2.18 \mathrm{~m}$. All three SMEs had a total of seven production employees $\left(E_{t}^{p}=7\right)$ although only five $\left(E_{t}^{p}=5\right)$ and six were available $\left(E_{t}^{p}=6\right)$ on the first and second working day after the flood water has receded. From the third day, all seven employees were available.

\subsection{SMEs in lightly and moderately flooded areas}

For all three simulation experiments, S1 to S3, the recovery profile of production level was approximately the same for both the SME located in the lightly and moderately flooded areas shown in Fig. 2. Recall, the production level is a function of a number of dynamic attributes as discussed in Section 3.1. In simulation experiment S1, post-flood, production was restored at a level of $1 \%$ at $t_{s}=402\left(t_{c}=16: 30\right.$ on day 9$)$, whereas in S2 and S3 production was restarted at a level of $4 \%$ at $t_{s}=207\left(t_{c}=15: 00\right.$ on day 5), i.e. 195 simulation ticks earlier than in S1 (see Fig. 3). This significantly earlier resumption in production was due to funds being available enabling quick acquisition of services (electricians, plumbers and IT specialists) without needing to wait for compensation from the insurance company. Furthermore, at $t_{s}=241$ ( $t_{c}=8: 00$ on day 6$)$, in S3 the SMEs allocated their own production employees to clean-up the premises despite having comprehensive insurance, whereas in S2 the SMEs elected to wait (2 days) for their insurance company to provide a cleaning service which attended at $t_{s}=393\left(t_{c}=12: 00\right.$ on day 9$)$. In S2, during this waiting period, SMEs allocated between 1 and 3 employees to work on post-flood behaviours and the remainder on production thus consuming raw materials, leading to a drop in its recovery profile. 


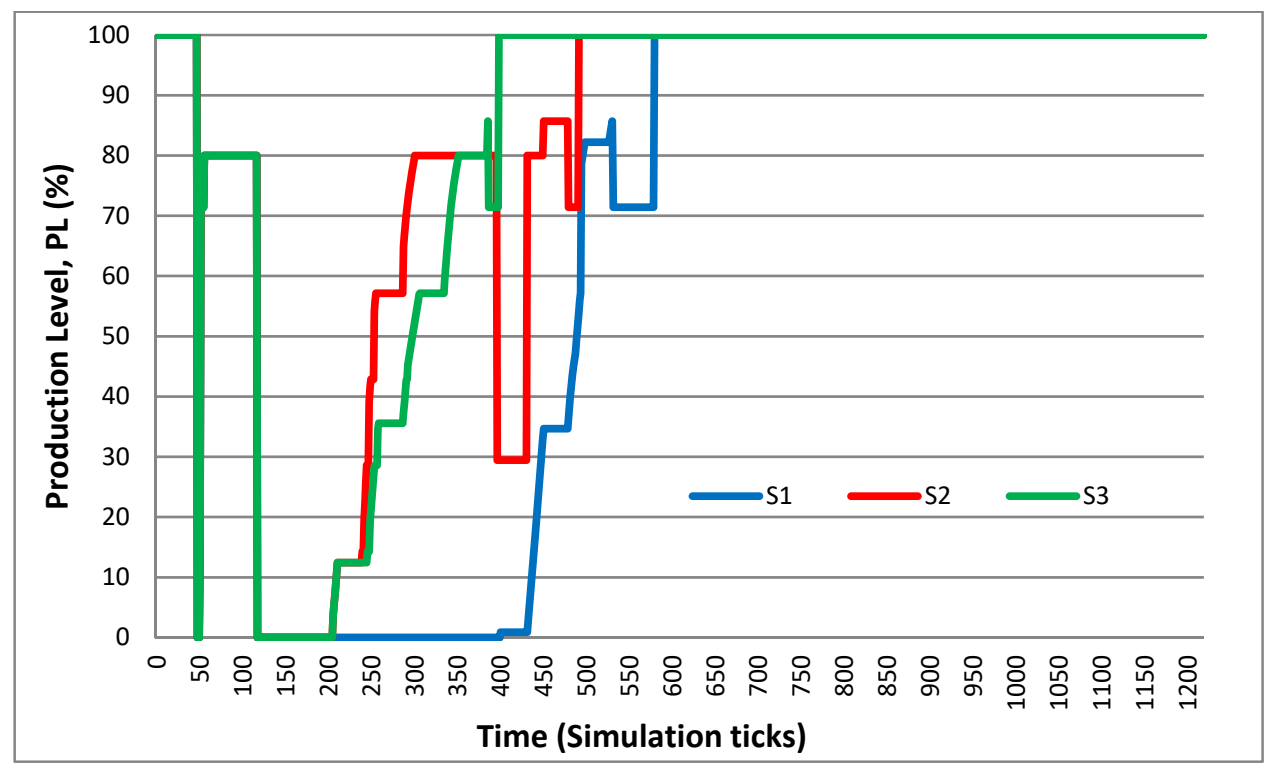

Figure 3: SMEs located in the lightly and moderately flooded areas.

As shown in Fig. 3, for S1, S2 and S3, production level was restored to 100\% in the SMEs located in the lightly and moderately flooded area at $t_{s}=581\left(t_{c}=10: 00\right.$ on day 13), $t_{s}=493$ $\left(t_{c}=14: 00\right.$ on day 11$)$ and $t_{s}=400\left(t_{c}=15: 30\right.$ on day 9$)$ respectively. Thus, in comparing S1 (SMEs with basic insurance and without funds available) with S3 (SMEs with comprehensive insurance and funds available but choosing to allocate their own employees to clean-up the premises rather than waiting for the insurance company provided cleaning service), it can be seen that full production was restored 90.5 hours (i.e. $581-400=181$ ticks) later.

\subsection{SME in severely flooded area}

In simulation experiment S1, for the SME located in the severely flooded area shown in Fig. 2, production $(P L=1 \%)$ was resumed post-flood at $t_{s}=402\left(t_{c}=16: 30\right.$ on day 9$)$, which coincides with the resumption of production of the SMEs located in the lightly and moderately flooded areas. However, as shown in Fig. 4, the production level of $100 \%$ was reached at $t_{s}=740\left(t_{c}=17: 30\right.$ on day 16$), 159$ simulation ticks later than for the SMEs located in the lightly and moderately flooded areas. Similarly, in S2 and S3, production resumed in the SME located in the severely flooded area at $t_{s}=433\left(t_{c}=8: 00\right.$ on day 10) and at $t_{s}=290$ $\left(t_{c}=8: 30\right.$ on day 7) respectively. That is, 226 and 83 simulation ticks later than in S2 and S3 of SMEs located in the lightly and moderately flooded areas. This delay in the resumption of production was due to the water depth reaching $2.18 \mathrm{~m}$ in the SMEs premises thus causing damage to raw materials, machines, IT equipment and documents that had been lifted-up preflood as preparatory behaviours when the EA alert was received. Also, the machines damaged required more time to be repaired due to the higher level of water reached in the SME's premises. Subsequently, in S2 and S3, production level reached $100 \%$ at $t_{s}=626\left(t_{c}=8: 30\right.$ on day 14$)$ and $t_{s}=589\left(t_{c}=14: 00\right.$ on day 13$)$ respectively. 


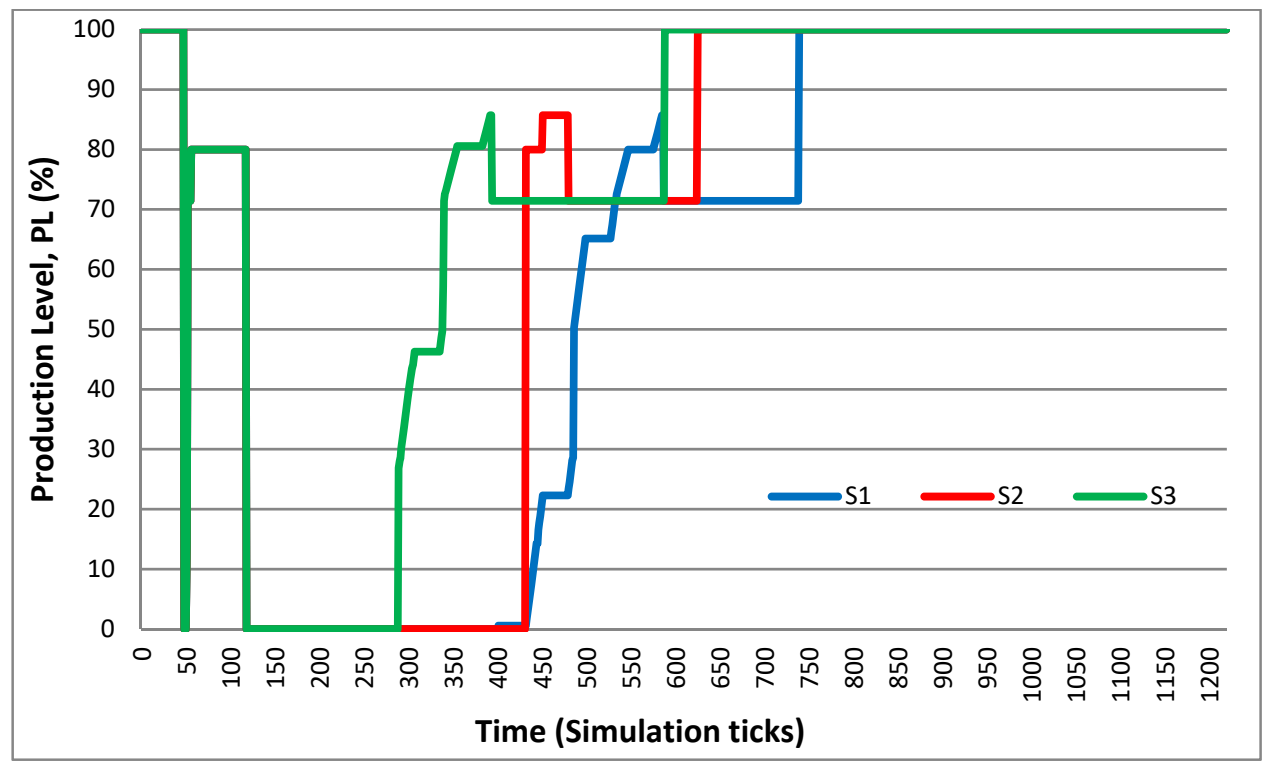

Figure 4: SME located in the severely flooded area.

\section{CONCLUSIONS}

This paper presented an overview of an ABMS approach that enables simulation-based investigations to be performed in terms of manufacturing SMEs resilience to flooding. Specifically, the paper discussed SMEs flood-disruption related attributes and behaviours and summarised an investigation into the effect of insurance coverage and availability of funds, combined with an SMEs decision on who to clean-up its premises, i.e. its own employees or a cleaning service. Three simulation experiments have been defined with each representing a different combination of insurance coverage, fund availability, and SMEs' decisions in terms of clean-up. Results indicate that SMEs with comprehensive insurance, and funds available, which choose to allocate their own employees to clean-up the premises (rather than waiting for the insurance company provided cleaning service) always recover more quickly than the other simulations considered regardless of the level of flooding experienced.

In term of future work, the ABMS framework will be further developed to model mutual aid partners to enable SMEs from the manufacturing sector to resume production earlier in the post-flood period than would otherwise be possible. Also, this framework will be developed to include modelling of SMEs from the retail sector.

\section{ACKNOWLEDGEMENT}

The authors thank colleagues from the School of Civil Engineering at the University of Leeds for providing inundation data from flood event simulation.

\section{REFERENCES}

[1] Pathak, S. \& Ahmad, M.M., Flood recovery capacities of the manufacturing SMEs from floods: A case study in Pathumthani province, Thailand. Int. J. Disast. Risk Re. 18, pp. 197-205, 2016. 
[2] Coates, G., Hawe, G., Wright, N. \& Ahilan, S., Agent-based modelling and inundation prediction to enable the identification of businesses affected by flooding. WIT Transactions on Ecology and the Environment, vol. 184, WIT Press: Southampton and Boston, pp. 13-22, 2014.

[3] Rhodes, C., Business statistics. House of Commons Library briefing paper, No. 06152, 2017.

[4] Lukács, E., The economic role of SMEs in world economy, especially in Europe. European Integration Studies, 4(1), pp. 3-12, 2005.

[5] White, S., Business Population Estimates for the UK and Regions, Department for Business, Energy \& Industrial Strategy: Sheffield, 2016.

[6] Crichton, D., Climate Change and its Effects on Small Businesses in the UK, Axa Insurance, 2006.

[7] Wedawatta, G., Ingirige, B. \& Amaratunga, D., Building up resilience of construction sector SMEs and their supply chains to extreme weather events. International Journal of Strategic Property Management, 14(4), pp. 362-375, 2010.

[8] Sullivan-Taylor, B. \& Branicki, L., Creating resilient SMEs: Why one size might not fit all. International Journal of Production Research, 49(18), pp. 5565-5579, 2011.

[9] Van Gils, A., Management and governance in Dutch SMEs. European Management Journal, 23(5), pp. 583-589, 2005.

[10] Jones, K. \& Ingirige, B. (eds), Extreme weather events and business continuity planning. CIB W70 International Conference in Facilities Management, 2008.

[11] Li, C., Coates, G., McGuinness, M. \& Johnson, N., Designing an agent-based model of SMEs to assess flood response strategies and resilience. International Journal of Bioengineering and Life Sciences, 9(1), pp. 7-12, 2015.

[12] Wedawatta, H., Resilience of construction SMEs to extreme weather events. PhD thesis, University of Salford, 2013.

[13] Chatterton, J., Viavattene, C., Morris, J., Penning-Rowsell, E.C. \& Tapsell, S.M., The Costs of the Summer 2007 Floods in England, Environment Agency: Bristol, 2010.

[14] Leinster, P., Flooding in England: A National Assessment of Flood Risk, Environment Agency: Bristol, 2009.

[15] Wedawatta, G. \& Ingirige, B., Resilience and adaptation of small and medium-sized enterprises to flood risk. Disaster Prevention and Management: An International Journal, 21(4), pp. 474-488, 2012.

[16] Dignum, V. \& Tick, C. (eds), Agent-based analysis of organizations: Formalization and simulation. Intelligent Agent Technology, 2007 IAT'07 IEEE/WIC/ACM International Conference on IEEE, 2007.

[17] Gilbert, N. \& Terna, P., How to build and use agent-based models in social science. Mind \& Society, 1(1), pp. 57-72, 2000.

[18] North, M.J. \& Macal, C.M., Managing Business Complexity: Discovering Strategic Solutions with Agent-Based Modeling and Simulation, Oxford University Press, 2007.

[19] Prasad, M.N. \& Chartier, D.A. (eds), Modeling organizations using agent-based simulations. workshop on agent simulation, applications, models, and tools, Citeseer, 1999.

[20] Johanson, L., Complexity modelling and application to policy research. Complex Systems Institute, University of North Carolina at Charlotte, 2015.

[21] Chappin, E.J., Dijkema, G.P., van Dam, K.H. \& Lukszo, Z. (eds), Modeling strategic and operational decision-making - an agent-based model of electricity producers. Proceedings of the 21st Annual European Simulation and Modelling Conference (ESM2007), St Julian's, Malta, 2007. 
[22] Clarke, K.C., Cellular automata and agent-based models. Handbook of Regional Science, eds M.M. Fischer \& P. Nijkamp, Springer: Berlin, pp. 1217-1233, 2014.

[23] Li, C. \& Coates, G., Design and development of an agent-based model for business operations faced with flood disruption. Complex Syst. Fundam. Appl., 90, p. 275, 2016.

[24] Alharbi, M. \& Coates, G., An agent-based modelling and simulation framework to assess small businesses' recovery from flooding. EMSS, Barcelona, Spain, 2017.

[25] Coates, G., Li, C., Wright, N. \& Ahilan, S., Investigating the flood responsiveness of small and medium enterprises using agent-based modelling and simulation. Flood Risk Management and Response, 173, 2016.

[26] Ordnance Survey, Britain's mapping agency 2018. Online. www.ordnancesurvey.co.uk. Accessed on: Feb. 2017.

[27] Morrissey, E., Environment Agency flood warnings: What you need to know 2016. Online. www.newground.co.uk/news-events-media/knowledge-centre/environmentagency-flood-warnings-what-you-need-to-know/. Accessed on: Feb. 2017.

[28] SEPA, Floodline Scottish Environment Protection Agency (SEPA) 2017. Online. www.sepa.org.uk/environment/water/flooding/floodline/. Accessed on: Feb. 2017.

[29] Coates, G. et al., A framework for organisational operational response and strategic decision making for long term flood preparedness in urban areas. WIT Transactions on the Built Environment, vol. 133, pp. 89-98, 2013.

[30] ABI, Responding to major floods, Association of British Insurers, 2016.

[31] Allianz, Loss adjuster frequently asked questions, 2018. 